\title{
Measurement of the Achilles tendon reflex for the diagnosis of lumbosacral root compression syndromes
}

\author{
R E RICO*, E J JONKMAN
}

From the TNO Research Unit for Clinical Neurophysiology, The Hague, The Netherlands

SUmmaRy The Hoffmann reflex and the Achilles tendon reflex were measured in a group of 194 subjects suspected of having a lumbosacral root compression syndrome. The Achilles tendon reflex was elicited manually with a metal hammer. There was a high correlation between the $\mathrm{H}-\mathrm{M}$ interval and the Achilles tendon reflex-M interval. The usefulness of the Achilles tendon reflex was evaluated in a selected sub-group of 61 patients with proven L5 or S1 root compression. Neither the H-reflex nor the Achilles tendon reflex appeared to be of any value in detecting L5 root compression. Both the H-reflex and the Achilles tendon reflex proved to be useful for diagnosis of S1 root compression syndromes, the latter being the more sensitive method.

Electromyography can be useful in the diagnosis of lumbosacral root compression syndromes. Examination of the involved muscles with concentric needle electrodes can reveal signs of denervation (fibrillations, positive sharp waves), a decrease in the number of motor units at maximum voluntary effort, and a high proportion of polyphasic potentials. Moreover, abnormalities can be expected in the late responses (reflexes and F-waves) recorded with surface electrodes. Often in the case of a nerve root lesion (due, for example, to a herniated disc) there will be no or only minor axonal damage. This implies that needle electromyography will reveal signs of denervation in only a relatively small number of patients. Compression of the nerve root, however, may cause local damage of the myelin sheath, resulting in a reduction in the proximal conduction velocity. Assuming a normal peripheral conduction velocity, the latent period of a monosynaptic reflex over the compressed roots will provide a measure of the abnormal proximal delay.

Many authors have emphasised the importance of reflex studies in the diagnosis of lumbosacral root compression syndromes. In particular the Hoffmann reflex evoked in the triceps muscle of the calf has attracted considerable attention. The mechanism of the H-reflex may be considered common knowledge and has been described elsewhere. ${ }^{1}$ Summarising,

"Present address: St. Elizabeth Hospital, Curaçao, Netherlands Antilles.

Address for reprint requests: Dr EJ Jonkman, Westeinde Hospital, Lijnbaan 32, 2512 VA The Hague, Netherlands.

Received 21 February 1982 and in revised form 30 April 1982

Accepted 14 May 1982 the H-reflex is a classical monosynaptic reflex, evoked electrically by stimulation of the IA-afferent fibres of the tibial nerve in the popliteal fossa. Via the dorsal root these afferent fibres reach the ventral horn of the spinal cord, where there is a monosynaptic connection with the alpha motoneurons which innervate the soleus muscle and the gastrocnemius muscle. Theoretically the pathway of this reflex is through the L5 and S1 roots. Measurement of the $\mathrm{H}$-reflex in the triceps muscles of the calf could thus provide some information about the presence of a compression syndrome at either one of these levels. Reflex parameters which can be measured are (a) the difference in time between the reflex response and the direct muscle response: "H-M interval" and (b) the amplitude, expressed as the ratio between the maximum obtainable reflex amplitude to the maximum direct muscle response $(\mathrm{H} / \mathrm{M})$. The latter parameter, however, is easily influenced by several factors, for example the position of the patient, degree of relaxation, etc. ${ }^{2}$ For this reason most authors nowadays are less inclined to use this ratio for the diagnosis of lumbosacral nerve root compression. The H-M interval proved to be a more reliable parameter, depending only on the patient's length, the nerve conduction velocity and the synaptic delay. In patients with a unilateral root compression syndrome, a left/right difference in the $\mathrm{H}-\mathrm{M}$ interval of $2 \mathrm{~ms}$ or more is considered significant. $^{3}$

Although the $\mathrm{H}$-reflex studies proved to be clinically useful, there are some practical disadvantages of this method: if properly done, the procedure is time-consuming and the patient must remain in an 
uncomfortable position; it requires considerable accuracy and patience on the part of the examiner to find the optimum position of the electrode for stimulation and the optimum intensity of the stimulus. The H-reflex as described above can be considered the electrical equivalent of the ankle jerk or Achilles tendon reflex, the only difference being that the $\mathrm{H}$-reflex bypasses the muscle spindles. To the best of our knowledge, measurement of the mechanically evoked Achilles tendon reflex has only been used for research purposes and not (yet) for the diagnosis of patients with an L5 or S1 root compression syndrome. ${ }^{4}$ The aim of this study was to determine the correlation between the H-reflex evoked in the triceps muscle of the calf and the Achilles tendon reflex in the same muscles, elicited by a simplified procedure. We also investigated whether the Achilles tendon reflex can contribute to the diagnosis of an L5 or S1 root compression syndrome.

\section{Material and methods}

A total of 194 subjects suspected on clinical grounds of having a unilateral lumbosacral root compression syndrome was studied. In one session the H-reflex, the Achilles tendon reflex and the maximum $\mathrm{M}$-response of the soleus muscle and the gastrocnemius muscle were measured on both sides. The reflexes were measured while the patient was lying in a prone position with the knee joints flexed at approximately $120^{\circ}$ and the ankle joints in a resting position of $100^{\circ}$ flexion. The tibial nerve was stimulated "monopolarly" in the popliteal fossa using as anode a large electrode on the contralateral leg. The stimulus frequency was $1 / \mathrm{s}$ or lower and the stimulus lasted 0.5 or $1.0 \mathrm{~ms}$. Recordings were made over the lateral part of the gastrocnemius muscle and over the soleus muscle; the average inter-electrode distance was $10 \mathrm{~cm}$. First the tibial nerve was stimulated using slowly increasing stimulus intensities in order to elicit a maximum H-reflex; subsequently the intensities were further increased to obtain a maximum M-response. For the Achilles tendon reflex the electrode positions were the same. The reflex was elicited manually with a metal hammer. Upon striking, the hammer made contact with a metal plate held against the Achilles tendon. This contact was used to trigger a digital delay unit, which made it possible to start the oscilloscope after a variable pre-set delay. Thus the measurement error of the latent period could be reduced to less than $0.2 \mathrm{~ms}$. The absolute latent periods (in $\mathrm{ms}$ ) and the maximum amplitudes (in $\mathrm{mV}$ ) of the $\mathrm{H}$-reflex, the Achilles tendon reflex (ATR) and the M-response were measured. For further processing only the $H-M$ interval and ATR-M interval were used. An asymmetry of 2 ms or more was considered to be abnormal.

For reasons already stated the amplitude ratios were not used. Moreover, in this study only a moderate correlation appeared to exist between the amplitude ratios for the $\mathrm{H}$-reflex and the Achilles tendon reflex.

\section{Results}

The correlation coefficients between the H-M interval and the ATR-M interval were computed for a sub-group of 136 patients. These coefficients (see table 1) show a good correlation between the two reflexes on both sides for the soleus muscle as well as the gastrocnemius muscle. However, an asymmetry of the H-M interval of 2 ms or more was not always accompanied by a significant asymmetry of the ATR-M interval or vice versa. Moreover an asymmetry over the soleus muscle was not always accompanied by an asymmetry over the gastrocnemius muscle.

To determine the usefulness of the different methods for diagnosis of an L5 or S1 root compression syndrome the results of the reflex measurements were evaluated in a more carefully selected sub-group. This sub-group consisted of 61 patients with a clinical unilateral L5 or S1 root compression syndrome, positive myelographic findings and a markedly herniated disc with unilateral compression of the L5 or S1 root, as confirmed upon surgery. In 27 subjects the L5 root was compressed and in 34 subjects, the $S 1$ root. These results were compared with findings for another sub-group of 28 patients in whom, despite the clinical signs, root compression could not be demonstrated either by myelography or surgically. All data are listed in tables $2-4$. It was found that neither the H-reflex nor the Achilles tendon reflex measured over either the soleus muscle or the gastrocnemius muscle is a sensitive method for the diagnosis of L5 root compression.

Even when "pathological" was defined as all those cases in which either the H-reflex or the Achilles tendon reflex was asymmetrical over one of the muscles under study, there was very little difference between patients with L5 lesions and the control group (see table 4, last column). On the other hand the $\mathrm{H}$-reflex as well as the Achilles tendon reflex appeared to be useful in detecting S1 root compression (with perhaps slightly better results for the gastrocnemius muscle than for the soleus muscle). In order to evaluate these two methods ( $\mathrm{H}$-reflex and Achilles tendon reflex), the findings for patients with proven S1 root compression were compared with those for the control group without proven root compression. Sensitivity, percentage of falsepositives and total accuracy were calculated. (All

Table 1 Correlation coefficients between $H$-M interval and $A T R-M$ interval $(N=194)$.

\begin{tabular}{lll}
\hline & Left & Right \\
\hline Musc soleus & 0.86 & 0.86 \\
Musc gastrocn & 0.84 & 0.87 \\
\hline
\end{tabular}


Table 2 Measurement of the Hoffmann reflex and Achilles tendon reflex $(A T R)$ over the soleus muscle in patients with L5 lesions, S1 lesions and controls. "Asymm.": left/right difference of the interval times $(H-M$ resp. $A T R-M) \geqslant 2 \mathrm{~ms}$. Last column: An asymmetry is indicated when the H-reflex and/or the ATR is asymmetrical.

\begin{tabular}{|c|c|c|c|c|c|c|}
\hline \multirow[t]{2}{*}{$M$ soleus } & \multicolumn{2}{|c|}{$H$-reflex } & \multicolumn{2}{|l|}{$A T R$} & \multicolumn{2}{|c|}{$H$-reflex $+A T R$} \\
\hline & symm & asymm & symm & asymm & symm & asymm \\
\hline \multirow{3}{*}{$\begin{array}{l}\text { L5 } \\
(\mathbf{N}=27) \\
\text { S1 } \\
(\mathbf{N}=34) \\
\text { Controls } \\
(\mathbf{N}=28)\end{array}$} & 24 & 3 & 23 & 4 & 23 & 4 \\
\hline & 15 & 19 & 9 & 25 & 8 & 26 \\
\hline & 24 & 4 & 25 & 3 & 23 & 5 \\
\hline
\end{tabular}

Table 3 Reflex measurements over the gastrocnemius muscle. For details see table 2.

\begin{tabular}{|c|c|c|c|c|c|c|}
\hline \multirow[t]{2}{*}{ M gastrocn. } & \multicolumn{2}{|c|}{$H$-reflex } & \multicolumn{2}{|l|}{$A T R$} & \multicolumn{2}{|c|}{$H$-reflex $+A T R$} \\
\hline & symm & asymm & symm & asymm & symm & asymm \\
\hline \multirow{3}{*}{$\begin{array}{l}\text { L5 } \\
(N=27) \\
S 1 \\
(N=34) \\
\text { Controls } \\
(N=28)\end{array}$} & 22 & 5 & 22 & 5 & 20 & 7 \\
\hline & 14 & 20 & 7 & 27 & 5 & 29 \\
\hline & 26 & 2 & 25 & 3 & 24 & 4 \\
\hline
\end{tabular}

Table 4 Combination of the data presented in tables 2 and 3. Last column: an asymmetry is indicated when an asymmetry is found over the soleus muscle and lor the gastrocnemius muscle.

\begin{tabular}{|c|c|c|c|c|c|c|}
\hline \multirow{2}{*}{$\begin{array}{l}M \text { sol + } \\
M \text { gastr. }\end{array}$} & \multicolumn{2}{|c|}{$H$-reflex } & \multicolumn{2}{|l|}{ ATR } & \multicolumn{2}{|c|}{$H$-reflex $+A T R$} \\
\hline & symm & asymm & symm & asymm & symm & asymm \\
\hline \multirow{3}{*}{$\begin{array}{l}\text { L5 } \\
(\mathrm{N}=27) \\
\text { S1 } \\
(\mathrm{N}=34) \\
\text { Controls } \\
(\mathrm{N}=28)\end{array}$} & 20 & 7 & 21 & 6 & 19 & 8 \\
\hline & 12 & 22 & 5 & 29 & 5 & 29 \\
\hline & 24 & 4 & 25 & 3 & 23 & 5 \\
\hline
\end{tabular}

data are listed in table 5). Measurement of the H-reflex over the soleus muscle and the gastrocnemius muscle did not appear to be a reliable method, since the results yielded a sensitivity of $55.9 \%$ for the soleus muscle and $58.8 \%$ for the gastrocnemius muscle with a total accuracy of $69 \cdot 4 \%$ and $74.2 \%$, respectively. Scoring as positive any difference in the $\mathrm{H}-\mathrm{M}$ interval of $2 \mathrm{~ms}$ or more over either the soleus muscle or the gastrocnemius muscle, the sensitivity was $64.7 \%$ and the total accuracy $74.2 \%$. Both the sensitivity and the total accuracy were higher for the Achilles tendon reflex. This applied for the measurements of the soleus muscle as well as the gastrocnemius muscle. The sensitivity for the gastrocnemius muscle was slightly higher than that for the soleus muscle.

The Achilles tendon reflex reached a maximum sensitivity of $85.3 \%$ with a total accuracy of $87.1 \%$ when the results for the soleus muscle and the gastrocnemius muscle were combined. There was no further improvement in sensitivity or total accuracy when the Achilles tendon reflex findings were combined with the H-reflex findings (table 5, last column).

\section{Discussion}

A significant asymmetry in the latent period for the H-reflex measured over the triceps muscle of the calf could be an indication of an L5 or S1 root compression syndrome. Notermans et $^{3} \mathbf{l}^{3}$ stated that compression of the L5 root could give rise to asymmetry of the H-reflex when measured over the gastrocnemius muscle and compression of the S1 root to asymmetry of the H-reflex when measured over the soleus muscle. Our results do not support this view. The $\mathrm{H}$-reflex could not in any way be shown to be a sensitive method for detection of L5 root compression. Like other authors ${ }^{5-12}$ we consider the H-reflex to be a useful contribution to the diagnosis of S1 root compression syndromes. In accordance with Hugon's review on the methodology of the $H$-reflex ${ }^{1}$ we expected to find that it would be sufficient to measure the reflex over the $M$ soleus only. However, our results did not confirm this since combining the findings for both calf muscles led to a higher sensitivity (table 5).

The high percentage of false-negative $\mathrm{H}$-reflex results $(35.3 \%)$ remains unsatisfactory. Comparison with the results of the others is barely possible. In some studies the sensitivity of the method used was not even mentioned. Sometimes the data for the $\mathrm{H}$-reflex are presented together with other EMG

Table 5 Sensitivity (S), percentage of false positives (FP) and total accuracy (TA) calculated for the patients with S1 lesions $(N=34)$ compared with the controls $(N=28)$.

\begin{tabular}{|c|c|c|c|c|c|c|c|c|c|}
\hline & \multicolumn{3}{|c|}{$H$-reflex } & \multicolumn{3}{|l|}{$A T R$} & \multicolumn{3}{|c|}{$H$-reflex $+A T R$} \\
\hline & $S$ & $F P$ & $T A$ & $s$ & $F P$ & $T A$ & $S$ & $F P$ & $T A$ \\
\hline $\begin{array}{l}\mathbf{M} \text { soleus } \\
\mathbf{M} \text { gastrocn. } \\
\mathbf{M} \text { soleus }+\mathbf{M} \text { gastrocn }\end{array}$ & $\begin{array}{l}55 \cdot 9 \\
58 \cdot 8 \\
64 \cdot 7\end{array}$ & $\begin{array}{r}14 \cdot 3 \\
7 \cdot 1 \\
14 \cdot 3\end{array}$ & $\begin{array}{l}69 \cdot 4 \\
74 \cdot 2 \\
74 \cdot 2\end{array}$ & $\begin{array}{l}73 \cdot 5 \\
79 \cdot 4 \\
85 \cdot 3\end{array}$ & $\begin{array}{l}10 \cdot 7 \\
10 \cdot 7 \\
10 \cdot 7\end{array}$ & $\begin{array}{l}80 \cdot 6 \\
83 \cdot 9 \\
87 \cdot 1\end{array}$ & $\begin{array}{l}76 \cdot 5 \\
85 \cdot 3 \\
85 \cdot 3\end{array}$ & $\begin{array}{l}17 \cdot 9 \\
14 \cdot 3 \\
17 \cdot 9\end{array}$ & $\begin{array}{l}79 \cdot 0 \\
85 \cdot 5 \\
83 \cdot 9\end{array}$ \\
\hline
\end{tabular}




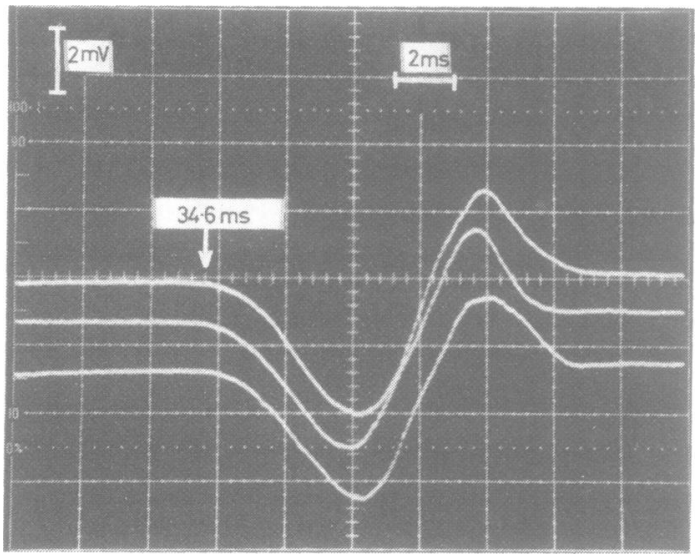

Fig 1 Registration of Achilles tendon reflex (3x). Trigger delay after stimulus $29.0 \mathrm{~ms}$. Note the good reproducibility of the reflex latency time $(34.6 \mathrm{~ms})$.

findings; the specific contribution of the reflex measurement could not therefore be estimated. ${ }^{313}$ Moreover we noted that some authors included needle myographic findings in their definition of an $\mathrm{S} 1$ syndrome. It is not surprising that in such studies a rather high sensitivity for the H-reflex measurements is reached. Most authors used the absolute latent period and not the $\mathrm{H} / \mathrm{M}$ interval. The upper limit of normally occurring asymmetry is sometimes fixed at $1.5 \mathrm{~ms}$ in contrast to the 2.0 seconds used by other authors. ${ }^{910}$

We could only compare our findings with part of the results of Fisher et al. ${ }^{11}$ Measuring the H-reflex in 16 patients with proven $S 1$ root compression they found a sensitivity of $43.8 \%$ with $56.3 \%$ falsenegatives. The number of false negatives was therefore even larger than in our study, but they also used the absolute latent period in their calculations. Evaluation of the findings for our 136 subjects showed a reasonable correlation between the $\mathrm{H}-\mathrm{M}$ interval and the ATR-M interval. Although in our experiments the stimulus for the Achilles tendon reflex was not quantified we still obtained reproducible responses, in particular as far as the latent period was concerned (fig 1). The examination is less unpleasant for the patient and is less timeconsuming than an H-reflex measurement. The Achilles tendon reflex also proved to be a more sensitive method for detecting $\mathrm{S} 1$ root compression syndromes (compared with the H-reflex) with a sensitivity of $85.3 \%$ and a total accuracy of $87 \cdot 1 \%$.

It is remarkable, when reviewing our results (table V) that we still found $14.3 \%$ false-positives for the H-reflex and $10.7 \%$ for the Achilles tendon reflex. It should be taken into account, however, that our control group consisted of subjects with lumbosacral radicular complaints, in whom anatomical root compression could not be demonstrated. A control group consisting of normal healthy individuals might perhaps have given fewer false-positive results. The measurement of Achilles tendon reflex appears to be a welcome addition to the electrophysiological diagnosis of S1 root compression syndromes. Combining this reflex with the results of $\mathrm{H}$-reflex measurements, however, does not increase the sensitivity. We therefore suggest that measurement of the Achilles tendon reflex, as described above, can replace $\mathrm{H}$-reflex measurement in the diagnosis of $\mathrm{S} 1$ root compression syndromes.

As there is no suitable monosynaptic reflex over the L5 root, needle myography remains the most suitable way to diagnose L5 compression syndromes. In patients with $\mathrm{S} 1$ root compression there are still reasons for needle myography: signs of denervation provide some information about the degree of axonal root injury and may contribute to the decision on whether to operate or not.

During this study it was noted that symmetry or asymmetry in briskness of the Achilles tendon reflex observed by the referring neurologist could not always be correlated with symmetry or asymmetry in the latency time of the electrically measured Achilles tendon reflex. In fact one should not expect to observe small differences in latency time as clinical phenomena. The extent to which clinical observations can be correlated with electrophysiological parameters remains an intriguing question. However, the procedure for such an investigation would differ from that used for the study presented here. For example, there would have to be a scoring scale for the clinically observed Achilles tendon reflex and interobserver reliability would have to be established. Moreover the electrophysiological data would have to include not only the latency time of the Achilles tendon reflex but also the amplitude, rise time, duration of the action potentials and possibly the mechanogram. Such a study is at present in the preparatory stage in our laboratory.

\section{References}

${ }^{1}$ Hugon M. Methodology of the Hoffmann reflex in man. In: Desmedt JE, ed. New Developments in Electromyography and Clinical Neurophysiology, (Basel, Karger 1973). 3:277-93.

${ }^{2}$ Landau WM, Clare MH. Fusimotor function, part IV: Reinforcement of the $\mathrm{H}$-reflex in normal subjects. Arch Neurol, 1964;10:117-22.

${ }^{3}$ Notermans SLH, Vingerhoets HM. The importance of the Hoffmann reflex in the diagnosis of lumbar root lesions. Clin Neurol Neurosurg, 1974;1:55-64.

${ }^{4}$ Dietrichson P, Sørbye R. Clinical method for electrical 
and mechanical recording of the mechanically and electrically elicited ankle reflex. Acta Neurol Scand 1971;47:1-21.

5 Baylan SP, Yu J, Grant AE. H-reflex latency in relation to ankle jerk, electromyographic, myelographic and surgical findings in back pain patients. Electromyogr Clin. Neurophysiol, 1981;21:201-6.

- Deschuytere J, Rosselle N. Diagnostic use of monosynaptic reflexes in L5 and S1 root compression. In: Desmedt, JE ed. New Developments in Electromyography and Clinical Neurophysiology, (Basel, Karger: 1973) 3;360-6.

${ }^{7}$ Guiheneuc P, Ginet J. The Hoffman reflex in lumbosacral root injuries. Electroencephalalogr Clin Neurophysiol, 1973;34:814-5.

${ }^{8}$ Descuns P, Collet M, Resche F, Lajat Y, Guiheneuc P, Ginet J. Intérêt du réflexe de Hoffmann dans l'explo- ration des lésions radiculaires lombo-sacrées d'origine discale. Neurochirurgie, 1973;19:627-37.

9 Braddom R, Johnson E. Standardization of $\mathrm{H}$-reflex and diagnostic use in S1radiculopathy. Arch Phys Med Rehabil, 1974;55:161-6.

${ }^{10}$ Schuchmann JA. H-reflex latency in radiculopathy. Arch Phys Med Rehabil, 1978;59:185-7.

" Fisher MA, Shivde AJ, Teixera C, Granier LS. Clinical and electrophysiological appraisal of the significance of radicular injury in back pain. $J$ Neurol Neurosurg Psychiatry, 1978;41:303-6.

${ }^{12}$ Aiello I, Rosati G, Serra G, Manca M. The diagnostic value of $\mathrm{H}$-index in $\mathrm{S} 1$ root compression. $J$ Neurol Neurosurg Psychiatry, 1981;44:171-2.

${ }^{13}$ Tonzola RF, Ackil AA, Shahani BT, Young RR. Usefulness of electrophysiological studies in the diagnosis of lumbosacral root disease. Ann Neurol, 1981, 9;305-8. 